\title{
Panobinostat synergizes with zoledronic acid in prostate cancer and multiple myeloma models by increasing ROS and modulating mevalonate and p38-MAPK pathways
}

\author{
F Bruzzese ${ }^{1}$, B Pucci ${ }^{2}$, MR Milone ${ }^{2}$, C Ciardiello ${ }^{1}$, R Franco ${ }^{3}$, MI Chianese ${ }^{1,7}$, M Rocco $^{1}$, E Di Gennaro ${ }^{1}$, A Leone ${ }^{1}$, A Luciano ${ }^{4}$, C Arra ${ }^{4}$, \\ D Santini ${ }^{5}$, M Caraglia $^{6}$ and A Budillon ${ }^{\star, 1,2}$
}

Patients with advanced prostate cancer (PCa) and multiple myeloma (MM) have limited long-term responses to available therapies. The histone deacetylase inhibitor panobinostat has shown significant preclinical and clinical anticancer activity in both hematological and solid malignancies and is currently in phase III trials for relapsed MM. Bisphosphonates (BPs), such as zoledronic acid (ZOL), inhibit osteoclast-mediated bone resorption and are indicated for the treatment of bone metastasis. BPs, including ZOL, have also shown anticancer activity in several preclinical and clinical studies. In the present report, we found a potent synergistic antiproliferative effect of panobinostat/ZOL treatment in three PCa and three MM cell lines as well as in a PCa ZOL-resistant subline, independently of p53/KRAS status, androgen dependency, or the schedule of administration. The synergistic effect was also observed in an anchorage-independent agar assay in both ZOL-sensitive and ZOL-resistant cells and was confirmed in vivo in a PCa xenograft model. The co-administration of the antioxidant $\mathrm{N}$-acetyl-L-cysteine blocked the increased reactive oxygen species generation and apoptosis observed in the combination setting compared with control or single-agent treatments, suggesting that oxidative injury plays a functional role in the synergism. Proapoptotic synergy was also partially antagonized by the addition of geranyl-geraniol, which bypasses the inhibition of farnesylpyrophosphate synthase by ZOL in the mevalonate pathway, supporting the involvement of this pathway in the synergy. Finally, at the molecular level, the inhibition of basal and ZOL-induced activation of p38-MAPK by panobinostat in sensitive and ZOL-resistant cells and in tumor xenografts could explain, at least in part, the observed synergism.

Cell Death and Disease (2013) 4, e878; doi:10.1038/cddis.2013.406; published online 24 October 2013

Subject Category: Cancer

Despite continuing efforts to develop effective and safe therapies for patients with locally advanced, recurrent, or metastatic prostate cancer ( $\mathrm{PCa})$, the overall survival, particularly in castration-resistant stage, has not improved significantly. ${ }^{1,2}$ Similarly, although novel antimyeloma agents have managed to significantly increase patients' overall survival in the past decade, multiple myeloma (MM) remains an incurable disease, and novel therapeutic approaches are urgently needed. ${ }^{3}$ Another major clinical challenge depends on the propensity of advanced PCa to metastasize to bone, which is primarily responsible for its effect on patient morbidity as well as mortality. ${ }^{4}$ On the other hand, bone marrow plasma cell overgrowth in MM induces osteolytic bone lesions, and several pieces of evidence suggest that the interaction between myeloma cells and the surrounding bone marrow microenvironment is critical for the progression of the disease. ${ }^{4,5}$

Nitrogen-containing bisphosphonates (N-BPs) such as zoledronic acid (ZOL) are currently used in oncological practice to reduce skeletal complications and pain, especially during myeloma and the metastatic phase of breast cancer and $\mathrm{PCa} .{ }^{6}$ Several reports suggest that N-BPs including ZOL

\footnotetext{
${ }^{1}$ Experimental Pharmacology Unit, Istituto Nazionale per lo Studio e la Cura dei Tumori ‘Fondazione Giovanni Pascale'-IRCCS, Napoli, Italy; ${ }^{2}$ Mercogliano Oncology Research Center (CROM), Istituto Nazionale per lo Studio e la Cura dei Tumori 'Fondazione Giovanni Pascale'-IRCCS, Napoli, Italy; ${ }^{3}$ Pathology Unit, Istituto Nazionale per lo Studio e la Cura dei Tumori 'Fondazione Giovanni Pascale'-IRCCS, Napoli, Italy; ${ }^{4}$ Animal Facility, Istituto Nazionale per lo Studio e la Cura dei Tumori 'Fondazione Giovanni Pascale'-IRCCS, Napoli, Italy; ${ }^{5}$ Department of Medical Oncology, University Campus Bio-Medico, Roma, Italy and ${ }^{6}$ Department of Biochemistry and Biophysics, Second University of Naples, Napoli, Italy

*Corresponding author: A Budillon, Experimental Pharmacology Unit, Istituto Nazionale per lo Studio e la Cura dei Tumori 'Fondazione Giovanni Pascale'-IRCCS, Via M. Semmola, 80131 Napoli, Italy. Tel: + 39081 5903292; Fax: + 39081 5903813; E-mail: a.budillon@ istitutotumori.na.it

${ }^{7}$ Current address: Laboratorio Patologia Clinica Bentivoglio, AUSL-BO Area Nord, Bologna, Italy

Keywords: panobinostat; zoledronic acid; prostate cancer; multiple myeloma; p38-MAPK

Abbreviations: PCa, prostate cancer; MM, multiple myeloma; ZOL, zoledronic acid; N-BP, nitrogen-containing bisphosphonate; FPP, farnesylpyrophosphate; GGPP, geranylgeranyl pyrophosphate; HDACi, histone deacetylase inhibitor; HSP27, heat shock protein-27; MAPK, mitogen-activated protein kinase; SRB, sulforhodamine B; MTT, 3-(4,5-dimethylthiazolyl-2)-2,5-diphenyl tetrazolium bromide; $\mathrm{IC}_{50}, 50 \%$ of cell growth inhibition; $\mathrm{Cl}$, combination index; DRI, dose reduction index; ELISA, enzymelinked immunosorbent assay; SDS-PAGE, sodium dodecyl sulfate-polyacrylamide gel electrophoresis; ECL, enhanced chemiluminescence; ROS, reactive oxygen species; NBT, nitroblue tetrazolium; TGD, tumor growth delay; H\&E, hematoxylin and eosin; TUNEL, terminal deoxynucleotidyltransferase (TdT)-mediated dNTP labeling; NAC, N-acetyl---cysteine; PARP, poly-(ADP-ribose)-polymerase; GGOH, isoprenolgeranyl-geraniol; uRap-1A, unprenylated Rap-1A

Received 02.7.13; revised 11.9.13; accepted 13.9.13; Edited by G Ciliberto
} 
exert potent antitumor effects in several cancer cell types including $\mathrm{PCa}$ and $\mathrm{MM}^{7}$ as well as in cancer patients. ${ }^{8-11}$

$\mathrm{N}$-BPs reduce bone loss in both osteoporosis and metastatic cancer by interfering with the mevalonate pathway inhibiting farnesylpyrophosphate (FPP) synthase. This inhibition causes FPP and geranylgeranyl pyrophosphate (GGPP) deprivation with a consequent inability to farnesylate and geranylate small GTPases of the RAS superfamily that eventually stop osteoclast-mediated bone resorption. .,12 $^{7}$

Notably, geranylgeranylated Ras and Rho family proteins are involved in different cellular processes that are critical for tumor development and progression, suggesting that the inhibition of protein geranylgeranylation is an important antitumor treatment strategy. ${ }^{13,14}$

Recently, our group has focused its interest on histone deacetylase inhibitors (HDACi), a new class of antitumor agent that demonstrated synergistic antitumor activity in combination with conventional anticancer agents and/or molecular target drugs. ${ }^{15-19}$ However, the clinical efficacy of HDACi, particularly in solid tumors, remains elusive, most likely because of the lack of understanding of the best context and combination regimen for their clinical use. ${ }^{20}$

The HDACi panobinostat (LBH589) has shown significant preclinical and clinical anticancer activity in both hematological and solid malignancies and is currently in phase III studies for relapsed $\mathrm{MM}^{21,22}$

In this study, we sought to preclinically define the potential anticancer effect of panobinostat in combination with ZOL in $\mathrm{PCa}$ and $\mathrm{MM}$ preclinical models. We demonstrated a synergistic antitumor activity of the two drugs both in vitro and in vivo. We also demonstrated that oxidative injury may play a functional role in the increased cell death observed in the combination treatment setting and that panobinostat may antagonize the acquired resistance to ZOL. Finally, at the molecular level, we present evidence that both the mevalonate and p38-mitogen-activated protein kinase (MAPK) pathways are involved in the mechanism of the synergistic antitumor effect.

\section{Results}

Panobinostat synergistically interacts with $\mathrm{ZOL}$ in $\mathrm{PCa}$ and MM cells. LNCaP, PC3, and DU145 PCa cells and RPMI8226, KMS12BM, and KMS21BM MM cells appeared responsive to the antiproliferative effect of panobinostat at $\mathrm{nM}$ concentrations, independently of p53 or KRAS status or any additional features ${ }^{23}$ (Table 1). ZOL effect varies among cell lines at $\mu \mathrm{M}$ concentrations, with LNCaP and RPMI8226 being the most resistant (Table 1). We investigated in all cell lines the effect of panobinostat/ZOL combination, exploring different treatment schedules, either simultaneously or sequentially (with a $24 \mathrm{~h}$ delay between the two agents), at equitoxic concentrations (50:50 cytotoxic ratio). We obtained consistent synergistic effects, with low combination indexes (Cls), calculated at $50 \%\left(\mathrm{Cl}_{50}\right)$ of cell lethality (highlighted in gray), in all cell lines independently from the schedule of administration (Table 2). The synergistic interaction between the two drugs was confirmed by the evaluation of the dose reduction indexes (DRIs), which represent the order of magnitude (fold) of dose reduction obtained for the $I_{50}\left(\mathrm{DRI}_{50}\right)$ in combination versus singledrug treatments (Table 2), and by soft agar assay (see below, Figure 1).

Panobinostat increases the ZOL-mediated antitumor effect in ZOL-resistant PCa cells. To investigate whether panobinostat can overcome resistance to ZOL, we took advantage of the ZOL-resistant DU145R80 subline developed in our laboratory from DU145 cells, ${ }^{24}$ and showing a sixfold higher $\mathrm{IC}_{50}$ of $\mathrm{ZOL}$ compared with parental cells (Table 1). Notably, even in these cells, which were also slightly more resistant to panobinostat (Table 1 and Supplementary Figure S1), we observed a clear synergism (Table 2). To confirm the synergistic effect observed thus far, a soft agar clonogenic assay was used to determine the effect of panobinostat and ZOL, alone or in combination, on DU145 (Figure 1a) and DU145R80 cells (Figure 1b). In DU145, panobinostat at $I C_{25}^{96 \mathrm{~h}}$ or $I \mathrm{IC}_{50}^{96 \mathrm{~h}}$ doses resulted in $\sim 37 \%$ and $45 \%$ colony formation inhibition, respectively. At same doses in DU145R80, we obtained a statistically significant $41 \%$ inhibition only with the higher dose of panobinostat, confirming that this cell line is slightly more resistant compared with DU145. Conversely, ZOL at $\mathrm{IC}_{25}^{96 \mathrm{~h}}$ or $\mathrm{IC}_{50}^{96 \mathrm{~h}}$ in DU145 resulted in $\sim 45 \%$ and $85 \%$ colony formation inhibition, respectively, whereas the DU145R80 were completely resistant. The concomitant panobinostat/ZOL treatment in DU145, at $\mathrm{IC}_{25}^{96 \mathrm{~h}}$ and $\mathrm{IC}_{50}^{96 \mathrm{~h}}$ of both agents, demonstrated $\sim 80 \%$ and $97 \%$ inhibition, respectively, whereas only the higher dose combination resulted in a significant 75\% inhibition in DU145R80.

These data clearly confirmed the significant potentiation of the antiproliferative effect when the two agents were combined in both ZOL-sensitive and ZOL-resistant cells.

The synergistic interaction of panobinostat/ZOL is mediated by ROS-induced apoptosis. To assess the mechanism of the synergistic antiproliferative effect, we evaluated apoptosis as well as reactive oxygen species (ROS) generation. As shown in Figure 2, the panobinostat/ ZOL combination induced a clear increase of apoptosis in PC3 cells (Figure 2a, upper panels) as compared with single agents, and this effect was paralleled by a significant increase in ROS levels (Figure $2 \mathrm{a}$, lower panels). Similar results were also obtained in DU145 and KMS12BM cells (Figures $2 \mathrm{~b}$ and $\mathrm{c}$ ) in which the ROS production induced by the combination was comparable with that induced by a $24 \mathrm{~h}$ exposure to $\mathrm{H}_{2} \mathrm{O}_{2}$ (positive control). Moreover, the co-administration of the antioxidant $\mathrm{N}$-acetyl-L-cysteine (NAC) antagonized the panobinostat/ZOL-mediated increase of both ROS and apoptosis in DU145 and KMS12BM cells (Figures $2 \mathrm{~b}$ and $\mathrm{c}$ ), confirming that oxidative injury plays a functional role in the enhanced lethality observed in the combination setting. Consistent with these findings, western blot analysis showed increased poly-(ADP-ribose)-polymerase (PARP) and caspase-3 cleavage in KMS12BM cells treated with the combination versus single agents, and this effect was completely antagonized by NAC (Supplementary Figure S2a). Notably, in the normal prostate epithelial cell line EPN, ${ }^{25}$ no synergistic interaction was observed in the 
Table 1 Prostate cancer (PCa) and myeloma multiple (MM) cell lines

\begin{tabular}{|c|c|c|c|c|c|c|}
\hline Cell lines & Origin & p53 & RAS & Additional features $^{a}$ & $\begin{array}{l}\text { Panobinostat } \\
\text { IC }_{50}(\mu \mathrm{M}) \\
96 \mathrm{~h} \pm \text { S.D. }\end{array}$ & $\begin{array}{l}\mathrm{ZOL} \\
\mathrm{IC} \\
96 \mathrm{~h} \pm \mathrm{S} . \mathrm{D}\end{array}$ \\
\hline $\begin{array}{l}\text { LNCaP } \\
\text { PC3 } \\
\text { DU145 }\end{array}$ & $\begin{array}{l}\mathrm{PCa} \\
\mathrm{PCa} \\
\mathrm{PCa}\end{array}$ & $\begin{array}{l}\text { WT } \\
\text { Null } \\
\text { Mut }\end{array}$ & $\begin{array}{l}\text { WT } \\
\text { WT } \\
\text { WT }\end{array}$ & $\begin{array}{l}\text { AR + ; androgen dep.; PTEN - mut; JAK1-mut } \\
\text { AR-null; androgen ind.; PTEN-null } \\
\text { AR +; androgen ind; PTEN - WT; CDKN2A-mut; MLH1-mut; RB1-mut; } \\
\text { STK11-null }\end{array}$ & $\begin{array}{l}0.05 \pm 0.02 \\
0.1 \pm 0.05 \\
0.05 \pm 0.014\end{array}$ & $\begin{aligned} 55 & \pm 6 \\
6.25 & \pm 0.0001 \\
22.99 & \pm 0.2\end{aligned}$ \\
\hline $\begin{array}{l}\text { RPMI8226 } \\
\text { KMS12BM }\end{array}$ & $\begin{array}{l}\mathrm{MM} \\
\mathrm{MM}\end{array}$ & $\begin{array}{l}\text { Mut } \\
\text { Null }\end{array}$ & $\begin{array}{l}\text { Mut } \\
\text { WT }\end{array}$ & $\begin{array}{l}\text { CDKN2C-mut; EGFR-mut } \\
\text { IL-6R, Cyclin D1, and BCL2 chromosomal gains; B7H1- and RS1 }\end{array}$ & $\begin{array}{l}0.036 \pm 0.005 \\
0.2 \pm 0.01\end{array}$ & $\begin{array}{r}40 \pm 3.2 \\
2.5 \pm 0.9\end{array}$ \\
\hline KMS21BM & MM & Null & WT & $\begin{array}{l}\text { IL-6R, Cyclin D1, and BCL2 chromosomal gains; B7H1- chromosomal } \\
\text { loss. }\end{array}$ & $0.031 \pm 0.05$ & $20 \pm 2.8$ \\
\hline DU145R80 & $\mathrm{PCa}$ & Mut & WT & DU145-derived ZOL-resistant cell line & $0.071 \pm 0.023$ & $145.90 \pm 1.8$ \\
\hline
\end{tabular}

${ }^{\mathrm{a}}$ For LNCaP, PC3, DU145, and RPMI8226, information has been derived from www.sanger.ac.uk. For KMS12BM and KMS21BM, information has been derived from Kaizu et $a l^{23}$

Table 2 Combination index $(\mathrm{Cl})^{\mathrm{a}}$ and dose reduction index (DRI) ${ }^{\mathrm{b}}$ values according to the different exposure of panobinostat/ZOL

\begin{tabular}{|c|c|c|c|c|c|c|c|c|c|}
\hline \multirow[t]{2}{*}{ Cell lines } & \multicolumn{3}{|c|}{ Panobinostat + ZOL } & \multicolumn{3}{|c|}{ Panobinostat $->$ ZOL } & \multicolumn{3}{|c|}{ ZOL $->$ panobinostat } \\
\hline & $\mathrm{Cl}_{50}( \pm S . D .)^{\mathrm{a}}$ & $\begin{array}{l}{ }^{\mathrm{b}} \mathrm{DRI}_{50} \\
\text { Panobin }\end{array}$ & $\begin{array}{l}\text { ( } \pm \text { S.D.) } \\
\text { ostat ZOL }\end{array}$ & $\mathrm{Cl}_{50}( \pm S . D .)^{\mathrm{a}}$ & $\begin{array}{c}{ }^{\mathrm{b}} \mathrm{DRI}_{50} \\
\text { Panobin }\end{array}$ & $\begin{array}{l}\text { ( } \pm \text { S.D.) } \\
\text { ostat ZOL }\end{array}$ & $\mathrm{Cl}_{50}( \pm \text { S.D. })^{\mathrm{a}}$ & $\begin{array}{c}{ }^{\text {b }} \mathrm{DRI}_{50} \\
\text { Panobin }\end{array}$ & $\begin{array}{l} \pm \text { S.D.) } \\
\text { ostat ZOL }\end{array}$ \\
\hline LNCAP & $0.48(0.07)$ & $8.58(3.56)$ & $3.2(0.71)$ & $0.59(0.12)$ & $8.6(2.7)$ & $2.4(0.44)$ & $0.52(0.09)$ & $3.63(1.23)$ & $6.4(5.27)$ \\
\hline PC3 & $0.63(0.098)$ & $4.15(1.76)$ & $2.96(1.5)$ & $0.55(0.2)$ & 7.05 (4.9) & $4.4(3.92)$ & $0.69(0.007)$ & 3.4 (1.97) & $3.5(2.22)$ \\
\hline DU145 & $0.31(0.03)$ & $5.75(0.32)$ & $7.28(0.98)$ & $0.49(0.08)$ & $3.11(0.04)$ & $4.06(4.96)$ & $0.48(0.0049)$ & $4.52(1.2)$ & $4.0 .5(1.04)$ \\
\hline RPMI8226 & $0.58(0.26)$ & $4.16(2.5)$ & 4.23 (1.96) & $0.48(0.07)$ & $5.03(0.24)$ & $3.48(0.83)$ & $0.89(0.43)$ & $5.32(4.34)$ & $1.72(0.59)$ \\
\hline KMS12BM & $0.67(0.1)$ & $0.13(0.1)$ & $1.68(0.1)$ & $0.46(0.09)$ & $0.14(0.2)$ & $1.76(0.24)$ & $0.61(0.19)$ & $0.12(0.4)$ & $3.15(0.3)$ \\
\hline KMS21BM & $0.18(0.2)$ & $4.1(0.1)$ & $16.32(5.42)$ & $0.63(0.55)$ & $2.49(3.5)$ & $22.96(0.2)$ & $0.76(0.21)$ & $2.7(0.33)$ & $2.61(2.1)$ \\
\hline DU145R80 & $0.69(0.14)$ & $1.65(0.07)$ & $3.9(0.84)$ & & & & & & \\
\hline
\end{tabular}

${ }^{\mathrm{a}} \mathrm{Cl}$ values (mean \pm S.D. from at least three separate experiments performed in quadruplicates) computed at $50 \%$ of cell kill $\left(\mathrm{Cl}_{50}\right)$ by $\mathrm{CalcuSyn}$ software (Biosoft, Cambridge, UK). Evaluation of Cls at equipotent doses (50:50 cytotoxic ratio) of the sequential exposure of the two agents panobinostat/ZOL. Simultaneous treatment (panobinostat $+\mathrm{ZOL}$ ), sequential exposure with $24 \mathrm{~h}$ delay, to either drug (panobinostat $\rightarrow Z O L$ or $Z O L \rightarrow$ panobinostat). Cls $<0.8$ indicated synergism and are highlighted in gray

${ }^{b}$ DRI values (mean \pm S.D. from at least three separate experiments performed in quadruplicates) represent the order of magnitude (fold) of dose reduction obtained for $\mathrm{IC}_{50}\left(\mathrm{DRI} \mathrm{I}_{50}\right)$ in combination setting compared with each drug alone

combination setting, although either panobinostat or ZOL alone induced an apoptotic effect (Supplementary Figure S2b).

Furthermore, to investigate whether the synergistic interaction between panobinostat and ZOL occurred via the mevalonate pathway, we evaluated apoptosis in the presence of geranyl-geraniol (GGOH) that overcomes the inhibition of FPP synthase induced by ZOL. ${ }^{26,27}$ As shown in Figure $2 b$, the addition of GGOH did not influence panobinostat effect, but it did inhibit ZOL-induced apoptosis and completely antagonized the synergistic effect in both DU145 and KMS12BM cells. Interestingly, the effect of GGOH was further improved, at least in DU145 cells, by concomitant treatment with NAC (Figure 2b). As a surrogate marker of N-BP uptake in our cancer models, we analyzed the expression of unprenylated Rap-1A (uRap-1A), a molecule that is shown to accumulate in cells as a result of the N-BP-mediated inhibition of FPP synthase and consequently of prenylation. The increased accumulation of URAP-1A was observed after $24 \mathrm{~h}$ of treatment with ZOL, or the combination, in both PC3 and DU145 cells (Figure 3c). Increased acetylation of histone H3 was used as control of panobinostat effect (Figure 3c).

All together, these findings suggested that oxidative injury plays a significant functional role in the enhanced lethality induced by the panobinostat/ZOL combination in PCa and MM cells, but not in normal prostate epithelial cells. In addition, the synergistic interaction between the two agents involves, at least in part, the mevalonate pathway.

The inhibition of the ZOL-induced p38-MAPK activation by panobinostat plays a critical role in the synergistic effects of the two drugs. To further assess the synergism at the molecular level, we evaluated several pathways in untreated or treated PC3 and DU145 cells by a multiplex phosphoprotein enzyme-linked immunosorbent assay (ELISA) assay. As shown in Figure 3, within $24 \mathrm{~h}$, both agents, among several pathways examined (data not shown), were able to modulate either AKT or ERK, depending on the cell line evaluated, with any interaction observed in the combination setting. In contrast, ZOL induced an $\sim 2$ - and 1.3-fold increase of p38-MAPK activation in PC3 and DU145 cells, respectively, compared with untreated cells (Figures $3 a$ and $b$ ), and this effect was completely abrogated by concomitant treatment with panobinostat. These results were confirmed by western blotting analysis that showed a sustained inhibition by panobinostat of the ZOL-induced p38-MAPK and heat shock protein-27 (HSP27) activation, the latter a well-known substrate of 
a
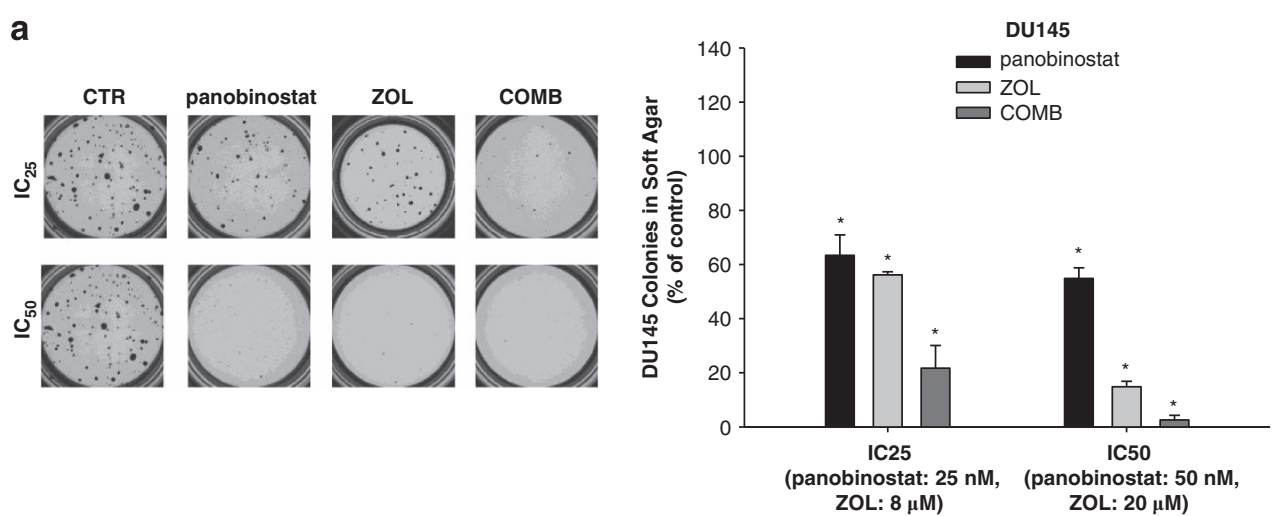

b

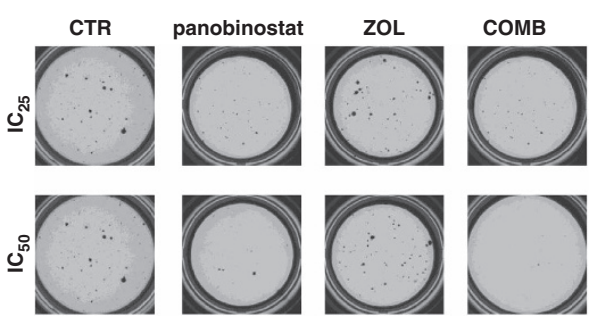

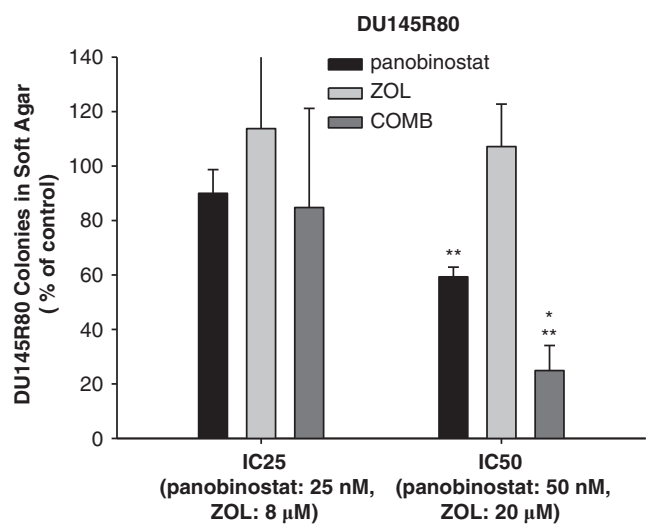

Figure 1 Synergistic inhibition of soft agar colony formation by the panobinostat/ZOL combination in DU145 and DU145R80 cell lines. Soft agar clonogenic assay in DU145 (a) and DU145R80 (b) cells that were untreated or treated with the indicated concentrations of panobinostat and ZOL alone and in combination. Colonies of $>100 \mu \mathrm{m}$ were scored by a colony counter. Left: images from a representative experiment; right: values expressed as the percentage of control are the mean \pm S.D. from at least three independent experiments. Statistically significant results are reported (DU145: ${ }^{*} P<0.001$ panobinostat or ZOL versus control and combination versus all other treatments; DU145R80: ${ }^{*} P<0.001$ combination versus control or ZOL; ${ }^{* *} P \leq 0,008$ panobinostat versus control and combination versus panobinostat)

p38-MAPK, in both PC3 and DU145 cells (Figure 3c and Supplementary Figure S3). Conversely, the basal activity of p38-MAPK and HSP27 was only slightly modulated by panobinostat. Although less evident, similar results were obtained in KMS21BM cells (Supplementary Figure S2).

Interestingly, in a recent report, we demonstrated that $\mathrm{ZOL}$ resistance in DU145R80 cells is mediated by a strong activation of the p38-MAPK-dependent pathway. ${ }^{24}$ In these cells, showing higher basal p38-MAPK phosphorylation compared with DU145, panobinostat completely downregulated p38-MAPK activation (Figure 3d). Finally, the role of p38-MAPK in the observed synergistic effect was additionally confirmed by demonstrating that the p38-MAPK inhibitor SB203580 increased apoptosis in ZOL-exposed DU145 cells (Supplementary Figure S4).

Altogether, these data showed that the inhibition of ZOLinduced p38-MAPK activation by panobinostat plays a critical role in the synergistic effects between the two drugs and confirmed previous findings that identified p38-MAPK as significantly involved in the sensitivity of cancer cells to the antitumor effect of ZOL. ${ }^{28-30}$

\section{In vivo activity of panobinostat/ZOL combined treatment} in a PCa xenograft model. To assess whether the synergistic antitumor effects demonstrated in vitro could be confirmed in vivo, we used a DU145 xenograft flank model.
As shown in Figure 4a, tumors in untreated mice grew rapidly and reached the end point size within 3 weeks of treatment. In the ZOL-treated group, the average tumor volume was slightly increased compared with untreated mice, whereas panobinostat treatment produced a measurable but modest tumor volume reduction compared with untreated mice (Figure 4a). However, a significant inhibition of tumor growth was found in the panobinostat/ZOL group (Figure 4a). Moreover, the resulting tumor growth delay (TGD) induced by the combination reached a peak of almost $40 \%$ compared with $20 \%$ in the panobinostat group, indicating that the rate of tumor growth in the control was almost 1.6-fold higher than in the combination setting (Figure 4b). The combined treatment was well tolerated, as shown by the maintenance of body weight (Figure $4 \mathrm{a}$, inset) and by the absence of other signs of acute or delayed toxicity.

Our analysis of hematoxylin and eosin (H\&E)-stained slides from the DU145 xenograft tumors showed an increase in the percentage of necrotic cells in the panobinostat/ZOL group compared with the untreated or the single-agent groups ( ${ }^{\star} P<0.001$; Figure 4c). Notably, a significant increase of apoptosis was also demonstrated by terminal deoxynucleotidyltransferase (TdT)-mediated dNTP labeling (TUNEL) assay in the panobinostat/ZOL group compared with control and single-agent groups ( ${ }^{\star} P<0.001$; Figure $4 \mathrm{c}$ ). The analysis of histone $\mathrm{H} 3$ acetylation, assumed as a pharmacodynamic 

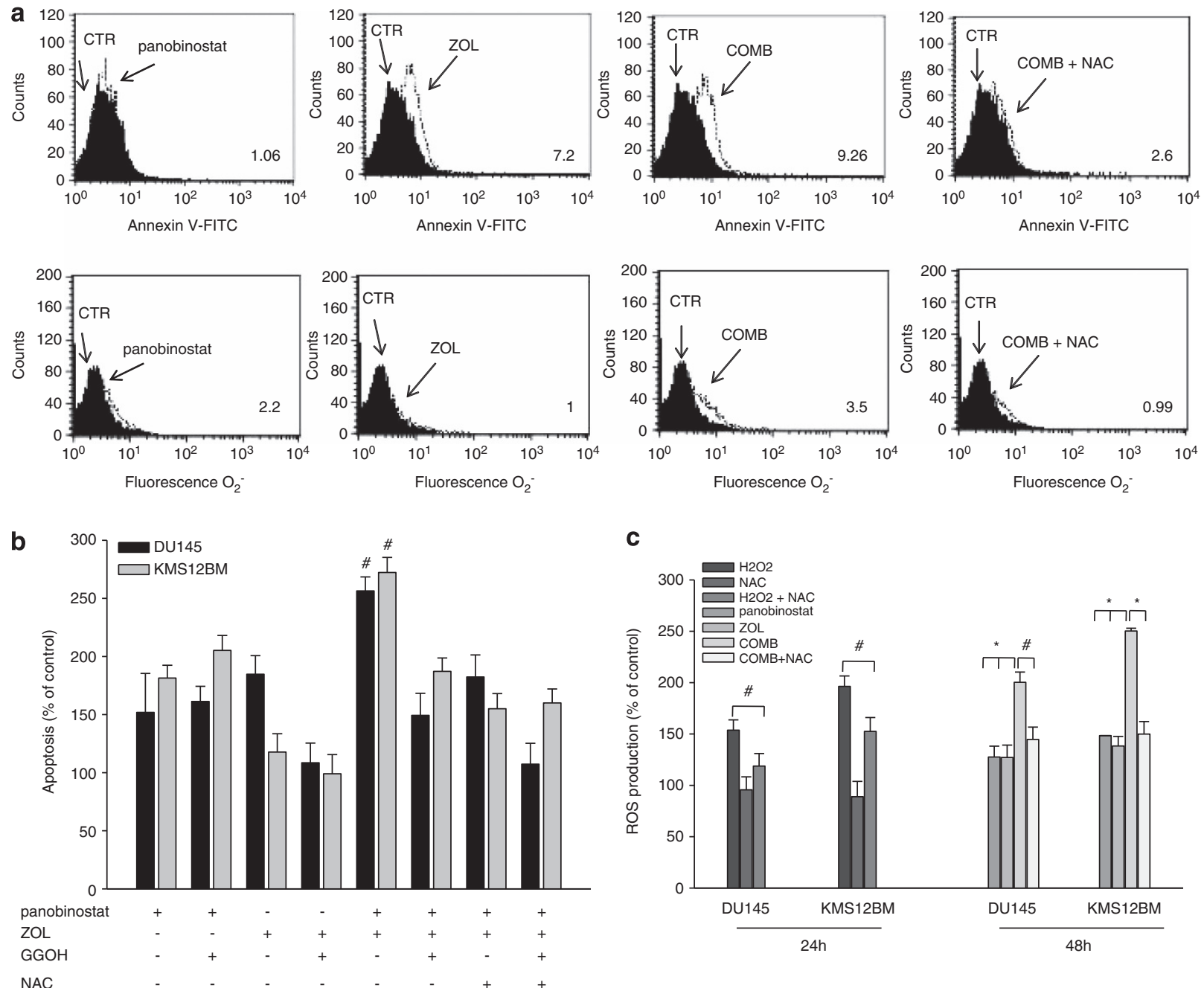

Figure 2 Correlation of enhanced ROS generation with induction of apoptosis shown by panobinostat/ZOL combination in PCa and MM cells. (a) PC3 cells untreated (CTR) or treated for $72 \mathrm{~h}$ with panobinostat and/or ZOL at the $\mathrm{IC}_{50}^{96 \mathrm{~h}}$ doses (panobinostat: $0.1 \mu \mathrm{M} ; \mathrm{ZOL}: 6.25 \mu \mathrm{M}$ ) (reflected by a rightward shift of the histogram) \pm the free radical scavenger NAC $(5 \mathrm{mM})$. Apoptosis, reflected by a rightward shift of the histogram, was evaluated by Annexin-V binding (upper panels), and the generation of reactive oxygen species (ROS) was evaluated by staining with hydroethidine followed by flow cytometry (lower panels). The fold inductions over untreated cells (CTR) are indicated for both apoptosis and ROS. (b) Apoptosis, evaluated by Annexin-V binding, in DU145 and KMS12BM cells untreated or treated for $48 \mathrm{~h}$ with panobinostat and/or ZOL at the IC $960 \mathrm{~h}$ doses (panobinostat: $0.05 \mu \mathrm{M}$ (DU145), $0.2 \mu \mathrm{M}$ (KMS12BM); ZOL: $17 \mu \mathrm{M}$ (DU145); $2.5 \mu \mathrm{M}$ (KMS12BM)) $\pm \mathrm{GGOH}(20 \mu \mathrm{M} 1 \mathrm{~h}$ before either treatment) to bypass the inhibition of FPP synthase by ZOL and/or NAC $(5 \mathrm{mM})$. The values, expressed as percentage of control, are the means \pm S.D. from at least three independent experiments $\left({ }^{\#} P<0.05\right.$ combination versus control and all other treatments). (c) Generation of ROS in DU145 and KMS12BM cells untreated or treated as indicated above, for the indicated points; cells treated for $24 \mathrm{~h}$ with $\mathrm{H}_{2} \mathrm{O}_{2}(2 \mathrm{mM})$ were the positive control. The values expressed as percentage of control are the means \pm S.D. from at least three independent experiments $\left({ }^{*} P<0.001 ;{ }^{*} P<0.05\right)$

marker of the deacetylase inhibitor effect, demonstrated a significant increase in both the panobinostat and panobinostat/ZOL groups as compared with control $\left({ }^{*} P<0.001\right.$ Figure 4c).

Finally, as shown in Figures $4 d$ and e, we confirmed by western blotting analysis that panobinostat also suppresses basal and ZOL-induced p38-MAPK and HSP27 activation in xenografted tumors.

\section{Discussion}

In the present study, we demonstrated that the combination of $\mathrm{N}-\mathrm{BP}$ ZOL with HDACi panobinostat induces synergistic antiproliferative and proapoptotic effects in PCa and MM cell lines, regardless of androgen dependency or the p53 and KRAS mutational status. Moreover, the effect of the panobinostat/ZOL combination in both PCa and MM cells was not schedule dependent, an observation that could be clinically relevant because a less stringent condition of drug administration would make this combination easily adaptable for clinical application.

We have also shown a clear synergism between the two agents in the DU145R80 ZOL-resistant PCa cell model, providing evidence for the first time that an HDACi can overcome resistance to ZOL. Finally, consistently with the in vitro data, the synergistic antitumor effect of panobinostat/ ZOL combination was also observed in vivo in a xenograft $\mathrm{PCa}$ model in nude mice. In detail, although we did not 
observe evidence for tumor regression, a significant inhibition of tumor growth was found in the panobinostat/ZOL versus single-agent treatments, together with more prominent histopathologic changes such as higher apoptotic activity and necrosis.

HDACis, including panobinostat, have been shown to be preclinically active in the suppression of PCa and MM growth, both in vitro and in vivo. ${ }^{21,31}$ Panobinostat, like other HDACi, is currently undergoing a large clinical development program in $\mathrm{MM}$ in combination regimens, ${ }^{21}$ and a clinical study in combination with docetaxel has recently been concluded in PCa patients. ${ }^{22}$ In addition to several preclinical studies, three randomized studies have demonstrated an anticancer benefit for ZOL that is distinct from its effect on bone in women receiving adjuvant endocrine therapy for early breast cancer. $^{8-10}$ Notably, a recent trial for $\mathrm{MM}$ demonstrated increased overall survival induced by ZOL, independently of the prevention of skeletal-related events, confirming a treatment benefit of this agent beyond bone health. ${ }^{11}$ However, a direct therapeutic activity of ZOL on tumors has been questioned because ZOL is readily excreted from the kidneys and cannot be maintained at high concentrations except in bone tissues. ${ }^{32,33}$ In humans, ZOL is administered once every 3 to 4 weeks with a peak serum concentrations in the range of $1-3 \mu \mathrm{M}$ that are detectable for few hours in the peripheral blood. ${ }^{34}$ The $\mathrm{IC}_{50}$ values of $\mathrm{ZOL}$ in our experimental cell models range from a minimum of $2.5 \mu \mathrm{M}$ to a peak of $55 \mu \mathrm{M}$, and the cells are exposed for $24 \mathrm{~h}$ up to few days, thus clearly above the human peak serum concentrations. However, by combining ZOL with panobinostat, we showed
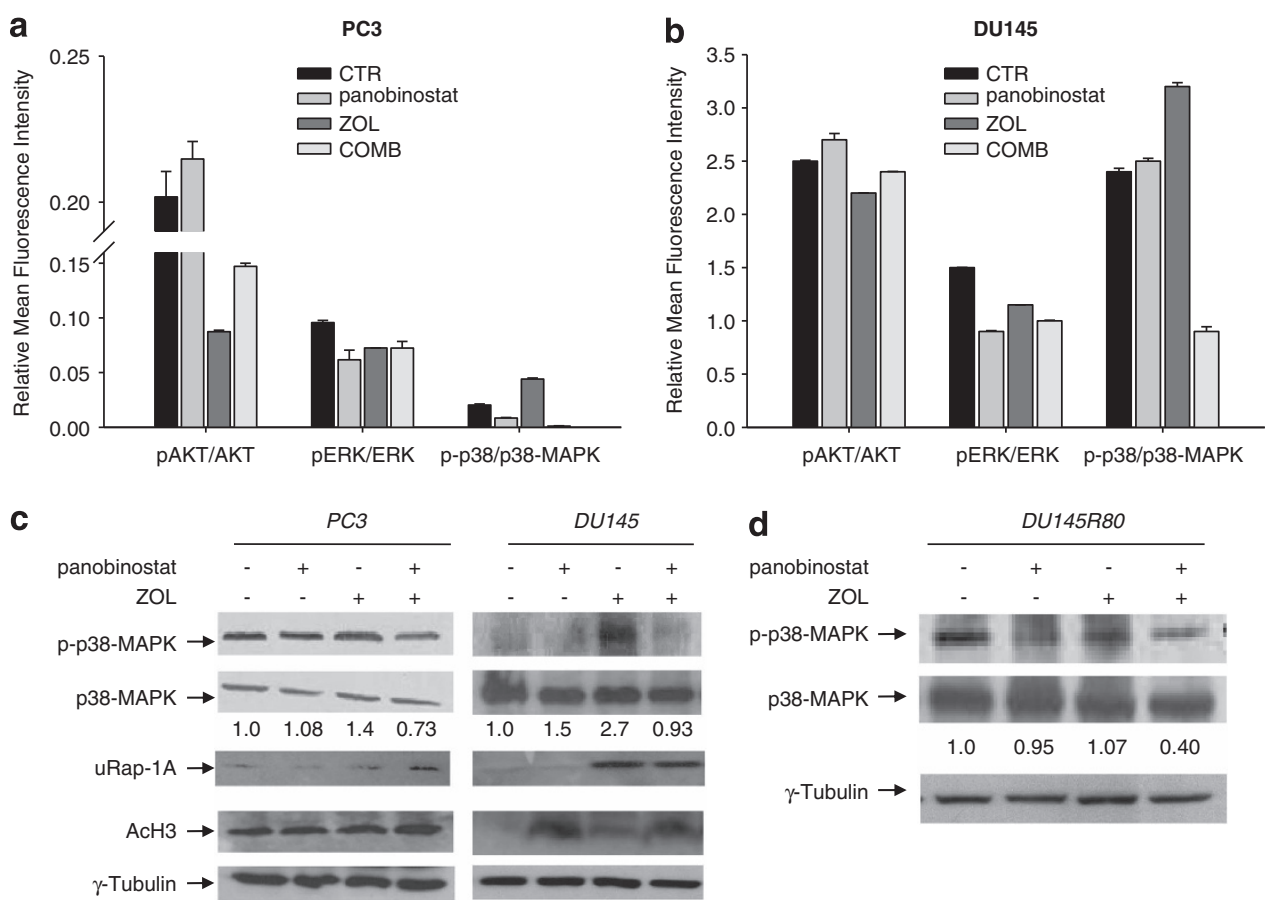

Figure 3 p38-MAPK involvement in the interaction between panobinostat and ZOL. Phosphoprotein ELISA-based multiplex immunoassay (see Materials and Methods) in PC3 (a) and DU145 (b) cells untreated (CTR) or treated for $24 \mathrm{~h}$ with panobinostat and/or ZOL at the IC 50 h $\mathrm{h}$ doses (panobinostat: $0.05 \mu \mathrm{M}, \mathrm{DU} 145 ; 0.1 \mu \mathrm{M}, \mathrm{PC} 3 ; \mathrm{ZOL}: 17 \mu \mathrm{M}$, DU145; $6.25 \mu \mathrm{M}$, PC3). (c) Western blotting analysis of phospho-p38-MAPK (p-p38-MAPK), p38-MAPK, unprenylated Rap-1A (uRap-1A), and acetylated histone H3 (AcH3) in PC3 and DU145 cells treated as indicated above. Extracts $(100 \mu \mathrm{g})$ were resolved by SDS-PAGE and immunoblotted with specific antibodies. The numbers below the panels indicate the ratio of phosphorylated to total p38-MAPK protein determined by densitometry. $\gamma$-Tubulin served as the control for equal protein loading. (d) Western blotting analysis of p-p38-MAPK and p38-MAPK in DU145R80 ZOL-resistant cells treated with panobinostat and/or ZOL at the IC 50 h doses of wt DU145 cells. Extracts (60 $\mu \mathrm{g}$ ) were resolved by SDS-PAGE and immunoblotted using specific antibodies. The numbers below the panels indicate the ratio of phosphorylated to total p38-MAPK protein determined by densitometry. $\gamma$-Tubulin served as the control for equal protein loading

Figure 4 Antitumor activity of panobinostat and ZOL on established PCa xenografts. DU145 cells $\left(7 \times 10^{6}\right)$ were s.c. injected into athymic mouse flanks as described in the Materials and Methods. When established tumors were palpable $\left(\sim 200 \mathrm{~mm}^{3}\right)$, mice were randomly assigned to four treatment groups (15 each): vehicles (CTR), panobinostat (15 mg/kg i.p.), ZOL (0.1 mg/kg i.p.), or both drugs (COMB), 5 days $/$ week for 2 weeks. (a) The mean \pm S.D. tumor volume measured at prespecified time points $(n=10) ;{ }^{*} P<0.001$. Inset, body weight measured three times/week. (b) Tumor growth delay (TGD) was determined as: \%TGD $=[(T-C) / C] \times 100$, in which $T$ and $C$ are the mean times expressed in days for the treated or control group, respectively, to reach a defined tumor volume (see Materials and Methods). (c) Paraffin-embedded tissue was prepared for each tumor. The necrotic areas were evaluated on H\&E-stained tissue and defined as the percentage of necrosis inner to tumoral nodule. The TUNEL assay and immunohistochemical analysis of histone $\mathrm{H} 3$ acetylation $(\mathrm{AcH} 3)$ were evaluated as described in the Materials and Methods and were scored semiquantitatively for the percentage of positive cells. Images were captured with a $\times 20$ objective on a light microscope. The group means were calculated for $n=5-7$ tumors per group. All of the data are presented as average \pm S.D. $\left({ }^{*} P<0.001\right)$. (d) Western blotting analysis of phospho-p38-MAPK (p-p38-MAPK), p38-MAPK, phospho-HSP27 (p-HSP27), and HSP27 in lysates from three different tumor from each treatment group (see Materials and Methods). Whole tumor protein lysates $(60 \mu \mathrm{g}$ ) were separated by $10 \%$ SDS-PAGE, transferred to nitrocellulose membranes, and immunoblotted with specific antibodies. $\gamma$-Tubulin immunoblotting ensured the equal loading of samples in each lane. (e) Densitometric analysis of western blotting from Figure 4d with NIH Image software. p-p38-MAPK and p-HSP27 levels were normalized to p38-MAPK and HSP27, respectively. Values, expressed as the ratio to the CTR group, are the mean \pm S.D. of the three different tumors for each group. ${ }^{\#} P<0.05 \mathrm{COMB}$ versus $\mathrm{ZOL}$ 

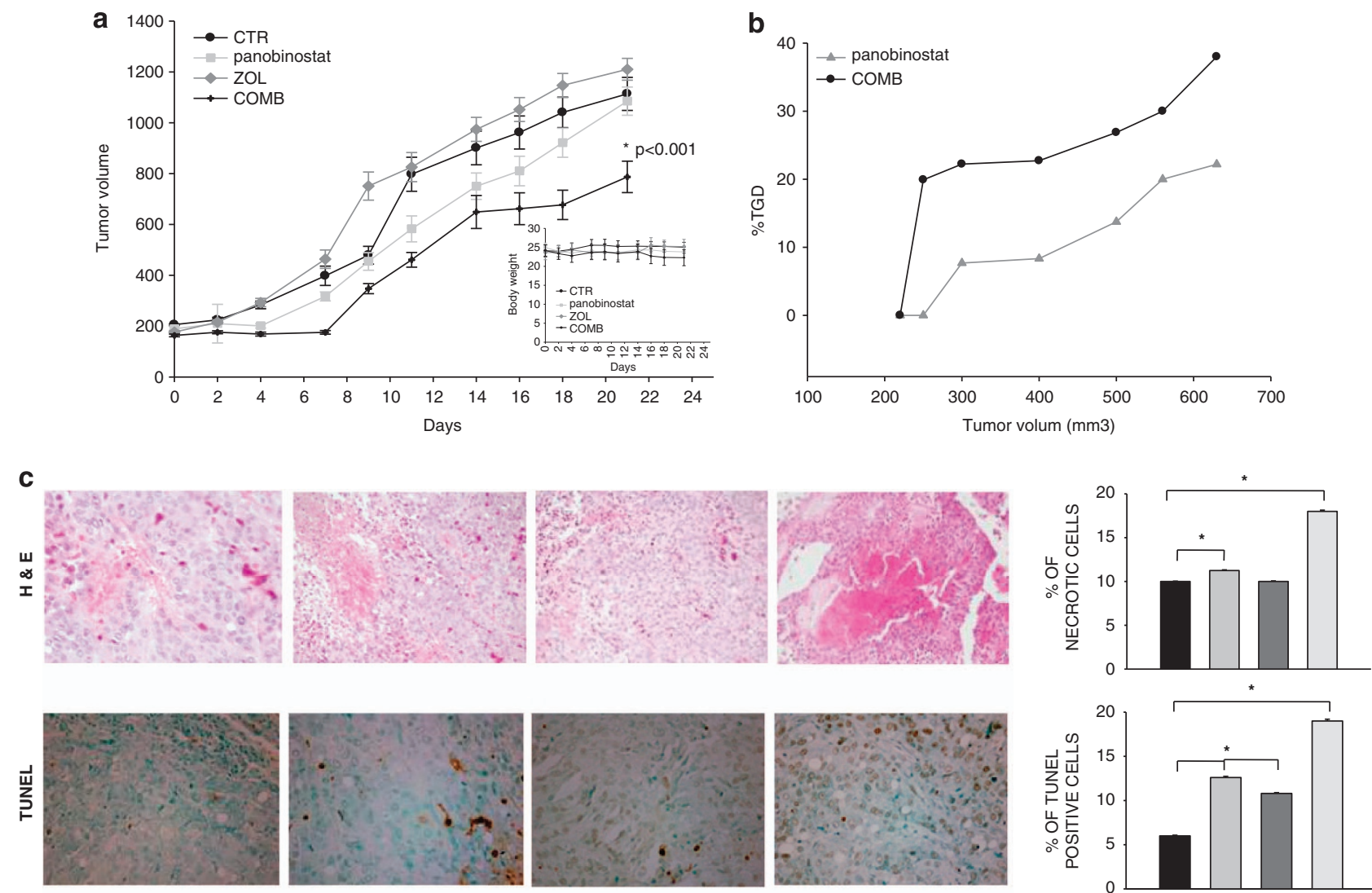

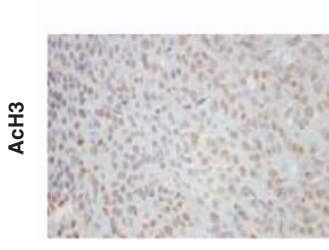

CTR
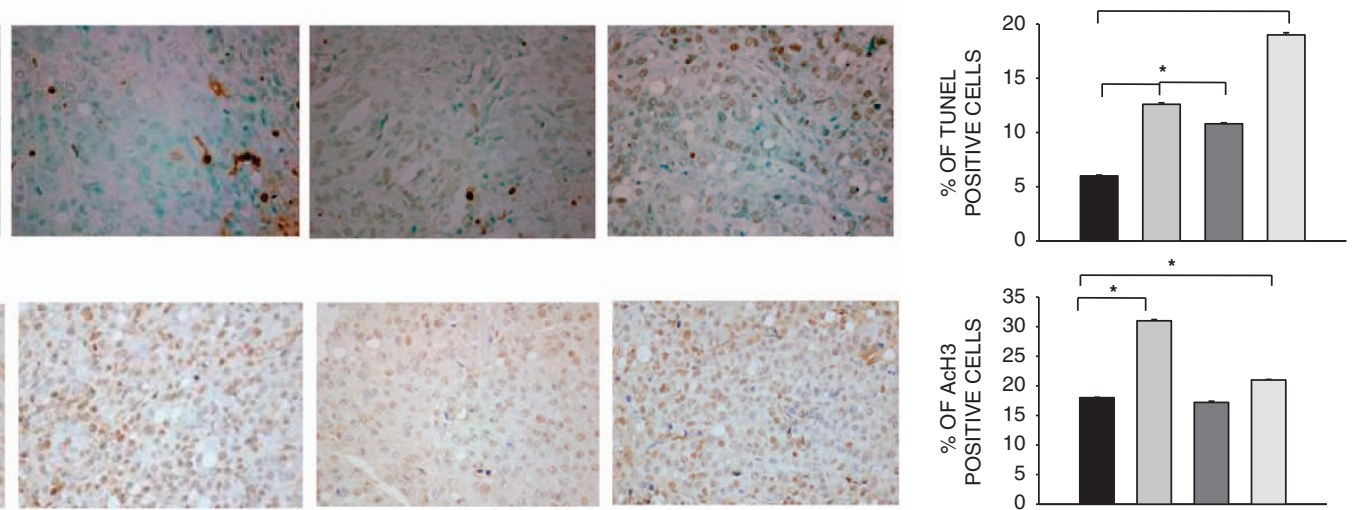

panobinostat

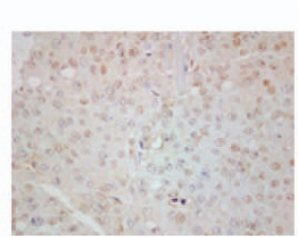

ZOL

d

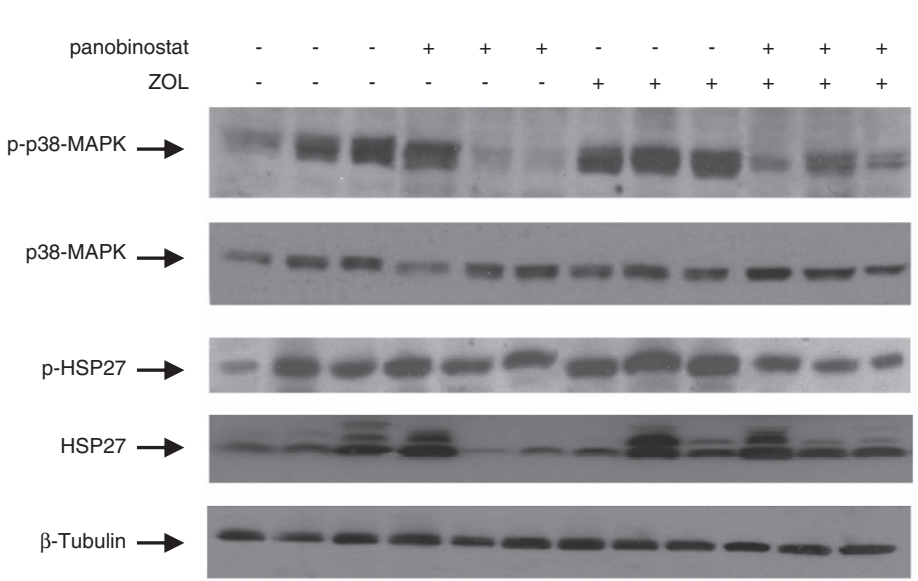

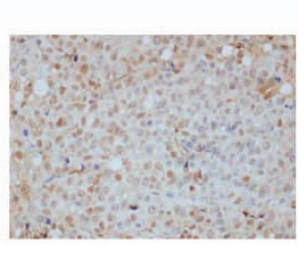

СомB
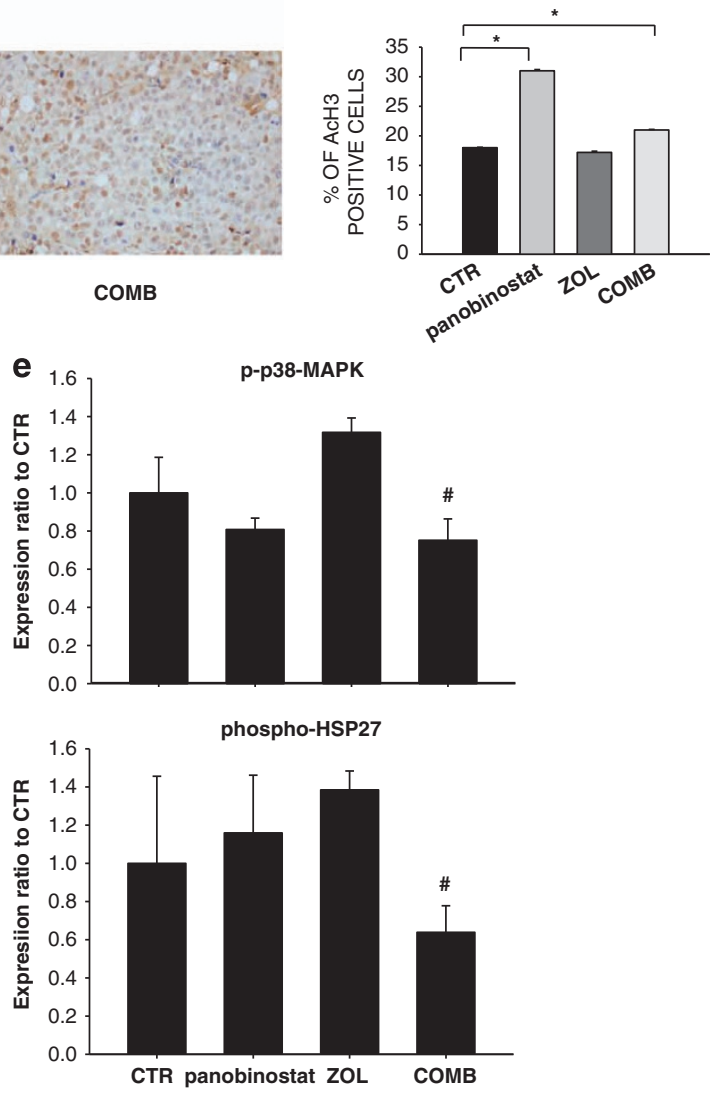
ZOL $\mathrm{IC}_{50}$ dose reduction from 1.7-fold to $>20$-fold versus single-drug treatment. Similarly, clear dose reductions for panobinostat $\mathrm{IC}_{50}$ were demonstrated in the combination setting in both PCa and MM cells. Moreover, we observed a statistically significant synergistic interaction between ZOL and panobinostat in the agar assay using $\mathrm{IC}_{25}$ doses of both agents. Furthermore, in the in vivo DU145 xenograft PCa model, we clearly demonstrated delayed tumor growth in the combination setting. Similarly, preclinical in vivo studies in tumor models, including $\mathrm{PCa}$, suggested that even low serum ZOL concentrations are sufficient to exert antitumor effect in peripheral tissues when combined with chemotherapeutics or molecular-targeted agents. ${ }^{7,34-37}$ Interestingly, we did not observe a significant apoptotic effect in the combination setting in the normal epithelial prostatic cell line EPN, suggesting that the synergistic interaction between the two agents could be confined to tumor cells. Indeed, we did not observe any increased loss of body weight or other signs of acute or delayed toxicity in vivo in the combinatory treatment.

Mechanistically, the apoptotic effect could be, at least in part, related to the parallel increase of ROS induced by the combination compared with the separate administration of the two drugs. Notably, the co-administration of NAC, which abolishes the increased ROS levels induced by panobinostat/ ZOL, also significantly reduced apoptosis, suggesting a causal role for oxidative damage in the induction of lethality. Indeed, neoplastic cells are more vulnerable to increased ROS because they function with a heightened basal level of ROS-mediated signaling, and the panobinostat/ZOL combination could lead to the accumulation of intracellular ROS to levels that exceed the cell's metabolic capabilities to maintain an acceptable physiological range and thereby induce apoptosis in cancer cells but not in normal cells.

We and others have shown that the generation of ROS represents an important mechanism by which high concentrations of HDACis exert their lethality. ${ }^{15,38-40}$ The mechanism by which this effect occurs involves alterations in the expression of redox-related genes and/or mitochondrial homeostasis. ${ }^{19,40}$ A direct effect of ZOL as a single agent on ROS generation has not been shown before; however, a recent report demonstrated that the increased intracellular ROS levels and the cytotoxic effect induced by ionizing radiation in cancer cells are enhanced by cotreatment with ZOL and that this effect is reduced by NAC. ${ }^{41}$ Notably, isoprenoids such as GGPP and FPP, which are inhibited in the cells treated with ZOL, are involved in the positive modulation of several nonsteroid isoprenoids (e.g., heme-A, coenzyme-Q10, and dolichols) that are related to antioxidant status. ${ }^{42}$ In light of these data, we can hypothesize that ZOL treatment may potentiate HDACiinduced oxidative stress by inhibiting the biosynthesis of antioxidant isoprenoids. This hypothesis is further supported by our results showing in both PCa and myeloma cell lines that the synergistic induction of apoptosis by panobinostat/ZOL is partially reverted by $\mathrm{GGOH}$, which has been shown to overcome the inhibition of FPP synthase induced by bisphosphonates, and is further inhibited by cotreatment with both $\mathrm{GGOH}$ and NAC. This finding indicates that the interaction between the two agents may occur via the mevalonate pathway. A previous study investigating the combination of ZOL and the HDACi vorinostat in vitro showed similar results. ${ }^{27}$
At the molecular level, we provided evidence that the inhibition by panobinostat of basal as well as ZOL-induced activation of p38-MAPK seems to play a critical role in the mechanism of the synergism in both sensitive and ZOLresistant cells. We have shown that ZOL induces, within $24 \mathrm{~h}$, a clear activation of p38-MAPK and of its effector HSP27 and that this effect is abrogated by concomitant treatment with panobinostat. Notably, we have recently demonstrated that the acquired resistance to ZOL in DU145R80 cells is mediated by $\mathrm{p} 38-\mathrm{MAPK}$ and can be reversed by a specific inhibitor of p38-MAPK. ${ }^{24}$ Interestingly, in this cell line, which expresses a higher level of activated p38-MAPK compared with parental DU145 cells, panobinostat completely inhibited p38-MAPK basal activation. Conversely, in DU145 and PC3 ZOLsensitive cells, the basal phosphorylation of p38-MAPK and HSP27 is slightly affected by panobinostat, suggesting that only an inappropriate activation of p38-MAPK pathway is targeted by HDACi. Notably, the inhibition by panobinostat of the ZOL-induced activation of both p38-MAPK and HSP27 was also observed in MM cells and in tumor specimens from PCa xenografted mice. Finally, the critical role of p38-MAPK in the synergism has also been indirectly confirmed by our finding that a p38-MAPK inhibitor potentiates ZOL-induced apoptosis.

Although the potential role of p38-MAPK activation in the resistance to $\mathrm{N}-\mathrm{BP}$ treatment has been previously proposed in breast cancer ${ }^{28}$ and osteosarcoma ${ }^{29,30}$ models, our study is the first to show a prosurvival role of p38-MAPK against ZOL in $\mathrm{MM}$ and in $\mathrm{PCa}$ both in vitro and in vivo and a suppressive antagonistic activity of panobinostat.

The increased activation of p38-MAPK has also been correlated with malignancy in several types of cancers, including PCa and MM, and several lines of evidence have suggested that, in cancer cells, p38-MAPK activation may facilitate proliferation, survival, invasion, and drug resistance. ${ }^{43,44}$ Indeed, in our previous study, we demonstrated that p38-MAPK activation mediated $\mathrm{ZOL}$ resistance in DU145R80 cells and also mediated the acquisition of a more aggressive phenotype characterized by epithelial-tomesenchymal transition (EMT), increased invasive capability, and the increased secretion of proinflammatory and proangiogenic cytokines as compared with parental cells. ${ }^{24}$

Interestingly, the isoprenoid $\mathrm{GGOH}$ in the mevalonate pathway has recently been shown to possess antioxidant, anti-inflammatory, antiapoptotic, and anticarcinogenic potential mediated at the molecular level by p38-MAPK inhibition. ${ }^{45}$ The latter study could further explain the activation of p38MAPK upon ZOL treatment as well as the correlation between apoptosis and oxidative stress found in our experimental model.

We have also shown that ZOL-induced p38-MAPK activation is paralleled within $24 \mathrm{~h}$ by the accumulation of unprenylated Rap-1A, a surrogate marker of the effect of ZOL, confirming that this agent targeted the mevalonate pathway in our cancer cell models.

In conclusion, to our knowledge, this study is the first to show the synergistic antitumor effect between ZOL and an $\mathrm{HDACi}$ such as panobinostat in both in vitro and in vivo $\mathrm{PCa}$ models and in an in vitro MM model. We also proposed a novel potential mechanism for the synergism between these agents 
relying on increased oxidative stress and dependent on crosstalk between the mevalonate and p38-MAPK pathways. The ability of panobinostat to synergize with and potentially overcome resistance to ZOL represents an attractive strategy that warrants clinical evaluation, even in combination with other agents, for the treatment of patients with bone-related cancers such as advanced PCa and MM.

\section{Materials and Methods}

Cell culture and cell viability assay. DU145, PC3, LNCaP, and RPMI8226 cells were purchased from American Type Culture Collections (Rockville, MD, USA). ZOL-resistant DU145R80 cells were obtained as previously described. ${ }^{24}$ EPN cells were kindly provided by Dr. D Tramontano (Department of Cellular and Molecular Biology and Pathology, University of Naples Federico II, Naples, Italy). ${ }^{25} \mathrm{KMS12BM}$ and KMS21BM MM cell lines were kindly provided by Dr. E Carbone (Department of Experimental and Clinical Medicine G Salvatore, University of Catanzaro Magna Graecia, Catanzaro, Italy). The cell viability was evaluated $96 \mathrm{~h}$ after treatment with sulforhodamine B (SRB) or by MTT (3(4,5-dimethylthiazolyl-2)-2,5-diphenyl tetrazolium bromide) assay, as previously described. ${ }^{15,16}$

The drugs and their preparation, all other reagents including antibodies, cell culture conditions, and other additional information are described in the Supplementary Materials and Methods.

In vitro drug combination studies. The drug combination studies were based on the concentration-effect curves generated as a plot of the fraction of unaffected (surviving) cells versus the drug concentration. ${ }^{15,16}$ Serial dilutions of equipotent doses of the two agents in combination (panobinostat and ZOL) were assessed. Synergism, additivity, or antagonism were quantified by determining the $\mathrm{Cl}$ calculated by the Chou-Talalay equation as described elsewhere. ${ }^{15,16}$ We assumed that $\mathrm{Cl}<0.9, \mathrm{Cl}=0.9-1.2$, and $\mathrm{Cl}>1.2$ indicate synergistic additive, and antagonistic effects, respectively. ${ }^{16}$ The DRI represents the measure of how much the dose of each drug in a synergistic combination may be reduced at a given effect level compared with the doses of each drug alone. ${ }^{16}$

Bio-Plex phosphoprotein detection assay. A multiplex ELISA-based immunoassay (Bio-Rad Lab Inc., Hercules, CA, USA) was used according to the manufacturer's instructions to evaluate the levels of protein phosphorylation on protein lysates from cells cultured and treated as indicated. Data acquisition and analysis were performed using Bio-Plex Manager software 3.0 (Bio-Rad). The phosphoprotein fluorescence intensities were normalized for the total protein expression intensities.

Protein extraction and western blotting. Cells grown and treated as indicated were collected, lysed, and separated by sodium dodecyl sulfatepolyacrylamide gel electrophoresis-polyacrylamide gel electrophoresis (SDS-PAGE); western blotting was performed as described elsewhere. ${ }^{18}$

Apoptosis assays. Annexin-V binding was identified by flow cytometry using Annexin-V-FITC staining, following the manufacturer's instructions as previously described ${ }^{15}$ (Becton Dickinson, San Jose, CA, USA)

Measurement of ROS generation. ROS generation was analyzed by flow cytometry using the ROS-sensitive dye hydroethidine, as described previously. ${ }^{15}$

Clonogenic agar assay. Cells were plated in 24-well, flat-bottomed plates using a two-layer soft agar system, as previously described. ${ }^{15}$ After $3 \mathrm{~h}$, the cells were treated with $\mathrm{ZOL}$ and/or panobinostat at the in vitro $\mathrm{IC}_{25}$ and $\mathrm{IC}_{50}$ of the drugs. The medium (with or without drugs) was replaced every 3 days. The colonies grew for 21 days and were then stained overnight with NBT (nitroblue tetrazolium), photographed, analyzed, and counted using Image-Pro-Plus (Immagini and Computer, Bareggio, Milano, Italy). Colonies of $>100 \mathrm{~mm}$ were scored as positive. All experiments were performed in triplicate.

In vivo xenograft assay. Xenograft experiments were conducted in female BALB/c nude mice (Charles River Laboratories, Milan, Italy) that were five weeks old. The mice received subcutaneous (s.c.) injections in the right flank of $7 \times 10^{6}$ DU145 cells/mouse in $200 \mu \mathrm{l}$ of a 1:1 mixed cell suspension and Matrigel. After tumor volumes reached $\sim 200 \mathrm{~mm}^{3}, \sim 1$ week after inoculation, a total of 60 xenografted mice were randomly assigned to four groups (15 each) to receive the maximum tolerated dose of panobinostat reported for animal studies ${ }^{31}(15 \mathrm{mg} / \mathrm{kg}$ daily i.p.), a ZOL dose equivalent to the approved clinical dose $(0.1 \mathrm{mg} / \mathrm{kg} 3$ days/ week i.p.), ${ }^{34}$ the combination of both agents, or control vehicles. Treatments were administered 5 days/week for 2 weeks. The body weight and tumor size (caliper) were measured three times/week, and the mice were killed when tumors reached an established cutoff volume of $\sim 1200 \mathrm{~mm}^{3}$. TGD was determined as $\%$ TGD = $[(T-C) / C] \times 100$, where $T$ and $C$ are the mean times expressed in days for the treated or control group, respectively, to reach a defined tumor volume. All experiments were approved in advance by the Institutional Animal Ethics Committee in accordance with the guidelines of the Italian Ministry of Health.

Histology, immunohistochemistry, and western blotting analysis from tumor samples. At the end of the experiment, tumors were then excised and split in two parts: one piece was frozen for biochemical studies and another piece was paraffin embedded for immunohistochemical analysis. Formalin-fixed and snap-frozen fragments of tumor specimens were paraffin embedded, and we evaluated necrosis by H\&E staining, apoptosis by (TUNEL, and histone $\mathrm{H} 3$ acetylation by immunohistochemistry, as reported previously. ${ }^{17}$ Study slides were examined by a single pathologist (RF) who was blinded to the final pathology interpretation. The group means were calculated with $n=5-7$ tumors per group. All data are presented as the average \pm S.D. Similarly, fragments from three different tumors from each treatment group were lysed and separated by SDS-PAGE, and western blotting was performed as described elsewhere. ${ }^{3}$ Proteins were transferred to nitrocellulose paper, immunoblotted with specific antibodies, and probed with the appropriate horseradish peroxidase-linked IgG. Immunoreactive bands were detected by enhanced chemiluminescence (ECL). Densitometric analysis was performed with the $\mathrm{NIH}$ Image software (National Institute of Health, Bethesda, MD, USA).

Statistics. The results of in vitro cell proliferation and of the soft agar clonogenic assay are expressed as the means for at least three independent experiments performed in quadruplicate; the S.D. is also indicated.

The results of apoptosis and the measurement of ROS generation by flow cytometry analysis were expressed as the means for at least three independent experiments ( \pm S.D.), and the statistical significance of differences was determined by two-sided Student's $t$-test. A probability of $<0.05$ was considered statistically significant. Representative results from western blotting, the Bio-Plex phosphoprotein assay, and immunohistochemistry from a single experiment are presented; additional experiments yielded similar results. All statistical evaluations were performed using Sigma Plot software (Systat Software Inc., San Jose, CA, USA).

\section{Conflict of Interest}

The authors declare no conflict of interest.

Acknowledgements. This work was partially supported by research grants to A Budillon from the nonprofit 'Associazione Italiana per la Ricerca sul Cancro' (AIRC IG 9332), the Italian Ministry of Health (FSN), and Novartis Pharmaceuticals (Basel, Switzerland). M Caraglia was supported by the Italian Ministry of Education, Research and University (PRIN 2009, Grant Number: 2009 EHW394). We are beholden to Dr. Alessandra Trocino from the National Cancer Institute of Naples for providing excellent bibliographic service and assistance.

1. Mahon KL, Henshall SM, Sutherland RL, Horvath LG. Pathways of chemotherapy resistance in castration-resistant prostate cancer. Endocr Relat Cancer 2011; 18: R103-R123.

2. Dayyani F, Gallick GE, Logothetis CJ, Corn PG. Novel therapies for metastatic castrate-resistant prostate cancer. J Natl Cancer Inst 2011; 103: 1665-1675.

3. Kuehl WM, Bergsagel PL. Molecular pathogenesis of multiple myeloma and its premalignant precursor. J Clin Invest 2012; 122: 3456-3463.

4. Coleman R, Gnant M, Morgan G, Clezardin P. Effects of bone-targeted agents on cancer progression and mortality. J Natl Cancer Inst 2012; 104: 1059-1067.

5. Anderson KC. The 39th David A. Karnofsky Lecture: bench-to-bedside translation of targeted therapies in multiple myeloma. J Clin Oncol 2012; 30: 445-452.

6. Saad F, Gleason DM, Murray R, Tchekmedyian S, Venner P, Lacombe L et al. Long-term efficacy of zoledronic acid for the prevention of skeletal complications in patients with metastatic hormone-refractory prostate cancer. J Natl Cancer Inst 2004; 96: 879-882. 
7. Caraglia M, Santini D, Marra M, Vincenzi B, Tonini G, Budillon A. Emerging anti-cancer molecular mechanisms of aminobisphosphonates. Endocr Relat Cancer 2006; 13: 7-26.

8. Gnant M, Mlineritsch B, Schippinger W, Luschin-Ebengreuth G, Postlberger S, Menzel C et al. Endocrine therapy plus zoledronic acid in premenopausal breast cancer. N Engl J Med 2009; 360: 679-691.

9. Coleman RE, Marshall H, Cameron D, Dodwell D, Burkinshaw R, Keane M et al. Breast-cancer adjuvant therapy with zoledronic acid. N Engl J Med 2011; 365: 1396-1405.

10. Coleman R, de Boer R, Eidtmann H, Llombart A, Davidson N, Neven P et al. Zoledronic acid (zoledronate) for postmenopausal women with early breast cancer receiving adjuvant letrozole (ZO-FAST study): final 60-month results. Ann Oncol 2013; 24: 398-405.

11. Morgan GJ, Davies FE, Gregory WM, Cocks K, Bell SE, Szubert AJ et al. First-line treatment with zoledronic acid as compared with clodronic acid in multiple myeloma (MRC Myeloma IX): a randomised controlled trial. Lancet 2010; 376: 1989-1999.

12. Rogers MJ, Crockett JC, Coxon FP, Monkkonen J. Biochemical and molecular mechanisms of action of bisphosphonates. Bone 2011; 49: 34-41.

13. van de Donk NW, Lokhorst HM, Nijhuis EH, Kamphuis MM, Bloem AC. Geranylgeranylated proteins are involved in the regulation of myeloma cell growth. Clin Cancer Res 2005; 11: 429-439.

14. Roy M, Kung HJ, Ghosh PM. Statins and prostate cancer: role of cholesterol inhibition versus prevention of small GTP-binding proteins. Am J Cancer Res 2011; 1: 542-561.

15. Bruzzese F, Rocco M, Castelli S, Di Gennaro E, Desideri A, Budillon A. Synergistic antitumor effect between vorinostat and topotecan in small cell lung cancer cells is mediated by generation of reactive oxygen species and DNA damage-induced apoptosis. Mol Cancer Ther 2009; 8: 3075-3087.

16. Di Gennaro E, Bruzzese F, Pepe S, Leone A, Delrio P, Subbarayan PR et al. Modulation of thymidilate synthase and p53 expression by HDAC inhibitor vorinostat resulted in synergistic antitumor effect in combination with 5FU or raltitrexed. Cancer Biol Ther 2009; 8: 782-791.

17. Di Gennaro E, Piro G, Chianese MI, Franco R, Di Cintio A, Moccia T et al. Vorinostat synergises with capecitabine through upregulation of thymidine phosphorylase. Br J Cancer 2010; 103: 1680-1691.

18. Bruzzese F, Leone A, Rocco M, Carbone C, Piro G, Caraglia M et al. HDAC inhibitor vorinostat enhances the antitumor effect of gefitinib in squamous cell carcinoma of head and neck by modulating ErbB receptor expression and reverting EMT. J Cell Physiol 2011; 226: 2378-2390.

19. Bianchi L, Bruzzese F, Leone A, Gagliardi A, Puglia M, Di Gennaro E et al. Proteomic analysis identifies differentially expressed proteins after HDAC vorinostat and EGFR inhibitor gefitinib treatments in Hep-2 cancer cells. Proteomics 2011; 11: 3725-3742.

20. Budillon A, Bruzzese F, Di Gennaro E. Histone deacetylase inhibitors in cancer therapy. Atta-ur-Rahman, M. Iqbal Choudhary (eds), Frontiers in Anti-Cancer Drug Discovery 2010; Bentham Science: The Netherlands. 978-1-60805-161-8.

21. Richardson PG, Mitsiades CS, Laubach JP, Hajek R, Spicka I, Dimopoulos MA et al. Preclinical data and early clinical experience supporting the use of histone deacetylase inhibitors in multiple myeloma. Leuk Res 2013; 37: 829-837.

22. Rathkopf D, Wong BY, Ross RW, Anand A, Tanaka E, Woo MM et al. A phase I study of oral panobinostat alone and in combination with docetaxel in patients with castrationresistant prostate cancer. Cancer Chemother Pharmacol 2010; 66: 181-189.

23. Kaizu KNH, Yata K, Otsuki T, Sugihara T, Amagasa T, Sato Y. Comparative genomic hybridization detected nonrandom chromosomal gains and losses in three pairs of sister myeloma cell lines establisced from bone marrow-and pleural effusion cells from the same patient. Oral Med Pathol 2004; 9: 103-111.

24. Milone MR, Pucci B, Bruzzese F, Carbone C, Piro G, Costantini S et al. Acquired resistance to zoledronic acid and the parallel acquisition of an aggressive phenotype are mediated by p38-MAP kinase activation in prostate cancer cells. Cell Death Dis 2013; 4: e641.

25. Sinisi AA, Chieffi P, Pasquali D, Kisslinger A, Staibano S, Bellastella A et al. EPN: a novel epithelial cell line derived from human prostate tissue. In Vitro Cell Dev Biol Anim 2002; 38 : 165-172.

26. Benford HL, Frith JC, Auriola S, Monkkonen J, Rogers MJ. Farnesol and geranylgeraniol prevent activation of caspases by aminobisphosphonates: biochemical evidence for two distinct pharmacological classes of bisphosphonate drugs. Mol Pharmacol 1999; 56: 131-140.

27. Sonnemann J, Bumbul B, Beck JF. Synergistic activity of the histone deacetylase inhibitor suberoylanilide hydroxamic acid and the bisphosphonate zoledronic acid against prostate cancer cells in vitro. Mol Cancer Ther 2007; 6: 2976-2984.
28. Merrell MA, Wakchoure S, Lehenkari PP, Harris KW, Selander KS. Inhibition of the mevalonate pathway and activation of p38 MAP kinase are independently regulated by nitrogen-containing bisphosphonates in breast cancer cells. Eur J Pharmacol 2007; 570: 27-37.

29. Kubo T, Shimose S, Matsuo T, Sakai A, Ochi M. Efficacy of a nitrogen-containing bisphosphonate, minodronate, in conjunction with a p38 mitogen activated protein kinase inhibitor or doxorubicin against malignant bone tumor cells. Cancer Chemother Pharmacol 2008; 62: 111-116.

30. Morii T, Ohtsuka K, Ohnishi H, Mochizuki K, Satomi K. Inhibition of heat-shock protein 27 expression eliminates drug resistance of osteosarcoma to zoledronic acid. Anticancer Res 2010; 30: 3565-3571.

31. Verheul HM, Salumbides B, Van Erp K, Hammers H, Qian DZ, Sanni T et al. Combination strategy targeting the hypoxia inducible factor-1 alpha with mammalian target of rapamycin and histone deacetylase inhibitors. Clin Cancer Res 2008; 14 : 3589-3597.

32. Skerjanec A, Berenson J, Hsu C, Major P, Miller WH Jr., Ravera C et al. The pharmacokinetics and pharmacodynamics of zoledronic acid in cancer patients with varying degrees of renal function. J Clin Pharmacol 2003; 43: 154-162.

33. De Luca A, Lamura L, Gallo M, Daniele G, D'Alessio A, Giordano P et al. Pharmacokinetic evaluation of zoledronic acid. Expert Opin Drug Metab Toxicol 2011; 7: 911-918.

34. Ottewell PD, Monkkonen H, Jones M, Lefley DV, Coleman RE, Holen I. Antitumor effects of doxorubicin followed by zoledronic acid in a mouse model of breast cancer. $J$ Natl Cancer Inst 2008; 100: 1167-1178.

35. Kuroda J, Kimura S, Segawa H, Kobayashi Y, Yoshikawa T, Urasaki Y et al. The thirdgeneration bisphosphonate zoledronate synergistically augments the anti-Ph + leukemia activity of imatinib mesylate. Blood 2003; 102: 2229-2235.

36. Caraglia M, Marra M, Leonetti C, Meo G, D'Alessandro AM, Baldi A et al. R115777 (Zarnestra)/Zoledronic acid (Zometa) cooperation on inhibition of prostate cancer proliferation is paralleled by Erk/Akt inactivation and reduced $\mathrm{Bcl}-2$ and bad phosphorylation. J Cell Physiol 2007; 211: 533-543.

37. Melisi D, Caputo R, Damiano V, Bianco R, Veneziani BM, Bianco AR et al. Zoledronic acid cooperates with a cyclooxygenase-2 inhibitor and gefitinib in inhibiting breast and prostate cancer. Endocr Relat Cancer 2005; 12: 1051-1058.

38. Ruefli AA, Ausserlechner MJ, Bernhard D, Sutton VR, Tainton KM, Kofler R et al. The histone deacetylase inhibitor and chemotherapeutic agent suberoylanilide hydroxamic acid (SAHA) induces a cell-death pathway characterized by cleavage of Bid and production of reactive oxygen species. Proc Natl Acad Sci USA 2001; 98: 10833-10838.

39. Dai YS, Xu J, Molkentin JD. The DnaJ-related factor Mrj interacts with nuclear factor of activated $\mathrm{T}$ cells $\mathrm{c} 3$ and mediates transcriptional repression through class II histone deacetylase recruitment. Mol Cell Biol 2005; 25: 9936-9948.

40. Ungerstedt JS, Sowa Y, Xu WS, Shao Y, Dokmanovic M, Perez G et al. Role of thioredoxin in the response of normal and transformed cells to histone deacetylase inhibitors. Proc Natl Acad Sci USA 2005; 102: 673-678.

41. Koto K, Murata H, Kimura S, Sawai Y, Horie N, Matsui T et al. Zoledronic acid significantly enhances radiationinduced apoptosis against human fibrosarcoma cells by inhibiting radioadaptive signaling. Int $J$ Oncol 2013; 42: 525-534.

42. Qi XF, Zheng L, Lee KJ, Kim DH, Kim CS, Cai DQ et al. HMG-CoA reductase inhibitors induce apoptosis of lymphoma cells by promoting ROS generation and regulating Akt, Erk and p38 signals via suppression of mevalonate pathway. Cell Death Dis 2013; 4: e518.

43. Cuenda A, Rousseau S. p38 MAP-kinases pathway regulation, function and role in human diseases. Biochim Biophys Acta 2007; 1773: 1358-1375.

44. Wagner EF, Nebreda AR. Signal integration by JNK and p38 MAPK pathways in cancer development. Nat Rev Cancer 2009; 9: 537-549.

45. Khan AQ, Khan R, Qamar W, Lateef A, Rehman MU, Tahir M et al. Geraniol attenuates 12-O-tetradecanoylphorbol-13-acetate (TPA)-induced oxidative stress and inflammation in mouse skin: Possible role of p38 MAP Kinase and NF-kappaB. Exp Mol Pathol 2013; 94: 419-429.

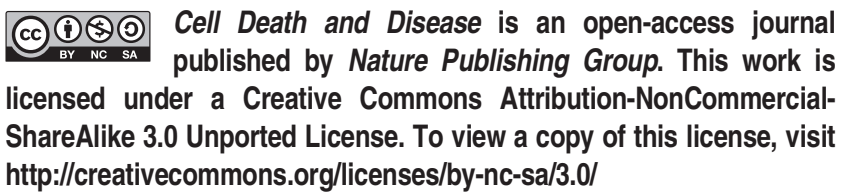
http://creativecommons.org/licenses/by-nc-sa/3.0/

Supplementary Information accompanies this paper on Cell Death and Disease website (http://www.nature.com/cddis) 